\title{
Pre- and post-menopausal differences in body fat distribution between Caucasians and South Asians: results from the Vitamin D, Food Intake, Nutrition and Exposure to Sunlight in Southern England (D-FINES) Study
}

\author{
A. L. Collins, K. Nosworthy, F. Hanjra, A. Osborn, A. Darling and S. A. Lanham-New \\ Faculty of Health and Medical Sciences, University of Surrey, Guildford GU2 7XH, UK
}

\begin{abstract}
Observations have shown that South Asians have a greater total and truncal (central) adiposity at a given BMI compared with Caucasian counterparts $^{(1)}$, corresponding to an increased risk of metabolic disease ${ }^{(2)}$. This finding has resulted in the suggestion that WHO cut-off values for BMI may not be an adequate basis for risk assessment in Asian populations ${ }^{(3)}$. However, many of these studies have focused on differences in men, with little consensus as to the specific ethnic differences in women. Furthermore, the influence of the menopause on fat distribution has been investigated in Caucasians and other ethnicities ${ }^{(4)}$ (e.g. African-American and East Asian) but not in South Asians. The whole-body assessment of individuals by dual-energy X-ray absorptiometry (DXA), as part of the D-FINES Study, allows an opportunity to explore ethnic differences in total and regional adiposity in a female cohort. In addition, the recruitment of both pre- and post-menopausal women offers insight into the influence of reproductive status on body composition in these ethnic groups.

Complete measurements of whole-body and regional-body composition were obtained in sixty-one South Asians and 216 Caucasian women living in southern England using DXA (Hologic QDR-4500; Hologic UK Ltd, Crawley, West Sussex, UK). Upper-body adiposity was calculated from the sum of the fat mass of the arms and trunk. Lower-body adiposity was calculated from the sum of both legs and upper-body:lower-body fat was calculated from the two variables. Summary descriptive and body composition data by ethnicity and reproductive status are shown in the Table.
\end{abstract}

\begin{tabular}{|c|c|c|c|c|c|c|c|c|}
\hline & \multicolumn{4}{|c|}{ Caucasians } & \multicolumn{4}{|c|}{ Asians } \\
\hline & \multicolumn{2}{|c|}{ Pre-menopause $(n$ 87) } & \multicolumn{2}{|c|}{ Post menopause $(n$ 129) } & \multicolumn{2}{|c|}{ Pre-menopause $(n$ 27) } & \multicolumn{2}{|c|}{ Post menopause (n 34) } \\
\hline & Mean & SD & Mean & SD & Mean & SD & Mean & SD \\
\hline Age (years) & 35.4 & 5.3 & $61.4^{*}$ & 4.5 & 41.1 & 6.3 & $59.4^{*}$ & 5.7 \\
\hline Weight (kg) & 67.9 & 11.9 & 69.7 & 12.4 & 65.9 & 12.9 & 70.4 & 15.5 \\
\hline BMI $\left(\mathrm{kg} / \mathrm{m}^{2}\right)$ & 25.1 & 4.4 & 26.5 & 5.0 & 26.3 & 5.1 & $29.4 * \dagger$ & 6.5 \\
\hline Total body fat $(\%)$ & 34.8 & 6.6 & $37.9^{*}$ & 6.0 & $37.9 \dagger$ & 5.7 & $42.7 * \dagger$ & 5.9 \\
\hline Trunk fat $(\%)$ & 32.3 & 8.1 & $36.6^{*}$ & 7.6 & $36.0 \dagger$ & 7.5 & $41.8^{*} \dagger$ & 6.8 \\
\hline Upper body fat (\%) & 33.5 & 8.1 & $37.8 *$ & 7.5 & $37.1 \dagger$ & 7.5 & $43.1^{*}$ & 6.9 \\
\hline Upper-body:lower-body fat & 1.47 & 0.41 & $1.83^{*}$ & 0.57 & 1.69 & 0.48 & 1.77 & 0.33 \\
\hline
\end{tabular}

Mean values were significantly different from those pre-menopause within ethnicity: $* P<0.05$. Mean values were significantly different from those for Caucasians of the same reproductive status: $\dagger P<0.05$.

Across the whole population (pre- and post menopause) total and regional adiposity was significantly higher in the South Asians compared with the Caucasians $(P<0.001)$. These differences remained after correcting for BMI in the whole population $(P \leq 0.014)$ as well as separately for pre-menopause $(P \leq 0.042)$ and post menopause $(P \leq 0.02)$. However, differences in regional adiposity between ethnic groups disappeared following correction for total body fat, suggesting regional adiposity in this cohort is a function of total adiposity. Nevertheless, these findings confirm the observation that South Asian women exhibit a greater adiposity at a given BMI.

When exploring differences between pre- and post-menopause groups, Caucasian post-menopausal women demonstrated significantly higher percentage trunk fat, upper-body fat and upper-body:lower-body fat compared with their pre-menopausal counterparts $(P \leq 0.02)$. Differences in upper-body fat and upper-body:lower-body fat remained even after correcting for total adiposity $(P \leq 0.04)$. In contrast, no differences in regional adiposity were seen between the pre- and post-menopausal South Asian women once corrected for total adiposity. This finding suggests that, although South Asian women may exhibit greater adiposity compared with Caucasians, more pronounced redistribution of fat centrally post menopause in the Caucasians could act to equalise differences in adipose-related health risk.

The D-FINES Study is funded by the FSA (NO5064). The views expressed are the authors' own.

1. Lear S, Humphries KH, Kohli S et al. (2007) Obesity (Silver Spring) 15, 2817-2824.

2. Misra A \& Vikram NK (2004) Nutrition 20, 482-491.

3. World Health Organization (2004) Lancet 363, 157-163.

4. Lovejoy JC, Champagne CM, de Jonge L et al. (2008) Int J Obes (Lond) 32, 949-958. 